\title{
Adoption et transfert de normes politiques : le cas des migrants maliens*
}

\author{
Lisa CHAUVET, économiste (IRD, DIAL) \\ Flore GUBERT, économiste (IRD, DIAL) \\ Sandrine MESPLÉ-SOMPS, économiste (IRD, DIAL)
}

\section{Introduction}

La circulation des hommes et des marchandises dans l'espace sahélien fait l'objet d'une attention croissante de la part de la communauté internationale. Elle est considérée comme déstabilisatrice, potentiellement génératrice de conflits et d'actes terroristes ${ }^{1}$. C'est d'ailleurs l'une des principales raisons de l'opération militaire française Barkhane au Mali qui vise à lutter contre les groupes armés djihadistes salafistes dans toute la région du Sahel. En outre, ces conflits politiques latents, combinés aux difficultés économiques des pays africains de la sous-région sont perçus comme accélérateurs des mouvements migratoires entre l'Afrique sub-saharienne d'un côté, et le Maghreb et l'Europe, de l'autre.

Dans ce contexte, le Mali tient une place centrale. Loin de se réduire à la circulation de combattants des mouvements extrémistes du nord du pays, les mouvements migratoires maliens sont historiquement importants et avant tout orientés vers les zones les plus riches d'Afrique de l'Ouest, notamment vers les côtes. De même, la présence de migrants maliens dans les pays européens tels que la France est ancienne et ne se cantonne pas aux

* Ce texte est publié dans Chauvet, L., Gubert, F., Jaulin T. et Mesplé-Somps S. (dir.) (2018) Migrants, acteurs des changements politiques en Afrique?, Deboeck supérieur, 240 p.

1. Pour une vision critique de l'approche française de la crise au Sahel, voir Guichaoua (2017). 
flux récents de réfugiés en provenance de cette région. Ainsi, estime-t-on que $11 \%$ des Maliens âgés de 18 ans et plus sont déjà partis en migration à l'étranger et que $20 \%$ ont actuellement un membre de leur famille en migration (Chauvet et al, 2013). La Côte d'Ivoire est le pays accueillant la plus grande communauté immigrée malienne. Environ $40 \%$ des migrants maliens y résident tandis que $65 \%$ des migrants de retour proviennent de ce pays. Viennent ensuite les autres pays limitrophes qui accueillent 15\% de la diaspora malienne (Sénégal, Burkina Faso, Niger, Mauritanie, Guinée), puis les autres pays d'Afrique (dont le Ghana et la Libye). Enfin, $16 \%$ des migrants maliens résident en France (mais seulement moins de $3 \%$ des migrants de retour en proviennent) tandis que les autres pays européens ou les États-Unis restent des destinations plus marginales².

L'objet de cet article est d'examiner les effets de la migration sur les institutions politiques maliennes. Comme souligné par Kapur et McHale (2005) et Kapur (2010), les effets de la migration sur l'économie politique des pays d'origine peuvent transiter par différents canaux. Tout d'abord, l'émigration, notamment des plus éduqués, prive le pays d'origine de ses individus les plus productifs, qui seraient le plus à même de revendiquer une amélioration des institutions politiques. Dans la terminologie de Hirschman (1970), cela réduit la protestation (voice). Par ailleurs, la modification de la composition socio-économique de la population induite par la migration peut altérer les préférences électorales et les équilibres politiques. Inversement, le fait qu'une partie de la population ait la possibilité de quitter le pays lui confère un pouvoir de négociation plus important qui lui permet d'exercer des pressions pour une meilleure gouvernance (exit dans la terminologie de Hirschman). De plus, les migrants peuvent, grâce à leur pouvoir économique ou à leurs réseaux dans le pays d'accueil, soutenir des groupes politiques spécifiques. La manne financière des migrants peut également être accaparée par les élites politiques à des fins de patronage par exemple. Enfin, les migrants peuvent acquérir en migration des normes sociales et politiques différentes qu'ils diffusent à leur communauté d'origine. Leur pouvoir économique, l’importance de leur réseau, voire leur participation

2. L'allocation par pays de destination de la diaspora malienne provient de Chauvet et al. (2013) tandis que celle des migrants de retour a été calculée par les auteures à partir de l'enquête EMOP 2013, représentative au niveau national. Ces estimations sont cohérentes avec celles issues du Recensement Général de la Population et de l'habitat (RGPH) du Mali réalisé en 2009, et avec le Recensement Administratif à Vocation d'État Civil (RAVEC). 
politique directe leur permettent alors d'agir sur les institutions politiques de leur pays d'origine. On parle alors de «diaspora channel». Il est également possible que les mécanismes liés à l'acquisition de normes politiques et au pouvoir économique des migrants continuent d'être à l'œuvre lorsque les migrants rentrent vivre dans leur pays d'origine. On parle alors de « return channel».

Ces mécanismes sont complexes et difficiles à appréhender. Jusqu’à présent, l'essentiel des travaux sur l'impact politique des migrants sur le pays d'origine a été mené de manière macro-économique. À titre d'exemple, Spilimbergo (2009) met en évidence que les pays dont le nombre d'étudiants à l'étranger dans des pays démocratiques est plus important connaissent une amélioration de leurs institutions démocratiques ; Docquier et al. (2016) montrent que l'émigration de personnes éduquées a un effet positif sur la qualité institutionnelle des pays d'origine. Quelques études ont également été menées sur des données individuelles. Dans le cas du Mexique, Pérez-Armendáriz et Crow (2010) et Pfütze (2012) mettent en évidence une amélioration du fonctionnement politique liée au phénomène migratoire. Les résultats de Barsbai et al. (2017) dans le cas de la Moldavie sont en cohérence avec le cas mexicain. Enfin, sur l'Afrique, l'essentiel des travaux existants fait l'objet des chapitres 5, 7 et 8 de cet ouvrage (Batista et al., 2017 ; Vari-Lavoisier, 2017 et Tuccio et Wahba, 2017) et confirme la relation positive existant entre le fonctionnement démocratique et la migration mise en évidence dans d'autres régions du monde.

Dans ce chapitre, nous tentons d'examiner l'influence de l'émigration internationale sur la vie politique malienne à travers l'acquisition de nouvelles normes politiques par les migrants et leur diffusion à la communauté d'origine. Tout d'abord, nous examinons dans quelle mesure l'expérience migratoire altère le regard porté par les migrants maliens sur les institutions démocratiques maliennes. Plus particulièrement, nous étudions les différences de perception des institutions maliennes entre les migrants et les non-migrants. Ensuite, nous analysons si les migrants de retour au Mali, du fait de leur expérience dans des pays aux institutions différentes, participent à la consolidation des institutions démocratiques maliennes. Ont-ils des opinions et des attitudes différentes concernant la politique nationale de leur pays d'origine ? Sont-ils à même d'influencer les personnes nonmigrantes et de modifier les pratiques politiques dans leur pays d'origine? 


\section{Contexte politique au Mali}

Les États d'Afrique de l'Ouest ont des institutions démocratiques fragiles. Les exemples d'élections occasionnant une alternance politique reconnue par l'ensemble des partis politiques et par la majorité de la population restent minoritaires. ${ }^{3}$ L'exemple du Mali illustre cette fragilité. La démocratie y est lancée en 1992, avec le renversement du régime militaire de Moussa Traoré par le chef des commandos parachutistes, Amadou Toumani Touré (ATT). Ce dernier met en place une nouvelle constitution et un code électoral qui permettent au pays de connaître une période de 20 ans durant laquelle des élections présidentielles, législatives et municipales sont organisées de manière régulière. Après deux mandats présidentiels d'Alpha Oumar Konaré (1992 et 1997), ATT est élu président de la République en 2002. Réélu en 2007, il est cependant renversé par un coup d'État en mars 2012, un mois avant l'organisation de nouvelles élections présidentielles auxquelles il ne se représentait pas. L'une des raisons de cette crise politique est l'insatisfaction d'une partie de l'armée et de la population concernant la gestion, par le gouvernement central, du conflit dans le nord du pays. Alors que la zone était déjà le théâtre de nombreuses rébellions depuis l'indépendance ${ }^{4}$, la fin du régime de Kadhafi en Libye, les mouvements extrémistes islamistes dans la sous-région et la porosité des frontières liée à la faiblesse de l'État malien ont intensifié ce conflit dès 20105. Au mouvement historique de rébellion touareg, le MNLA (Mouvement National pour la Libération de l'Azawad), se sont adjoints des groupes armés salafistes djihadistes régionaux tels que Ansar Dine et le Mujao (Mouvement pour l'unicité et le djihad en Afrique de l'Ouest). Courant 2012, ces derniers prennent possession des principales villes du Nord (Gao, Tombouctou) et avancent vers Bamako. Cette percée est interrompue par l'armée malienne grâce à l'appui de l'armée française. Après une année de transition, un nouveau scrutin présidentiel a été organisé au cours de l'été 2013, qui a conduit à l'élection d'Ibrahim Boubakar Keïta, ancien Premier ministre entre 1994 et 2002 et président de l'Assemblée nationale entre 2002 et 2007.

3. Les élections au Ghana depuis la fin des années 1990 ou au Sénégal depuis les années 2000 sont des exceptions notables.

4. Cf. Boilley, 1999 ; Bourgeot, 1996, 2013.

5. Cf. Guichaoua, 2014. 


\section{L'acquisition de normes politiques en migration : le cas des Maliens vivant en France et en Côte d'Ivoire}

L'expérience migratoire expose les individus à des contextes sociopolitiques qui diffèrent de leur pays d'origine. Les migrants peuvent alors devenir vecteurs de changement en diffusant à leur communauté d'origine les normes sociales et politiques acquises en migration. Mais ce processus de transfert de normes à la communauté d'origine nécessite que les migrants aient vu leurs propres référentiels normatifs altérés par l'expérience migratoire. Levitt (1998) souligne toutefois que les migrants ne sont pas tous perméables aux nouvelles idées et normes sociales. Le processus d'acquisition de normes dépend des interactions qu'ils entretiennent avec leur société d'accueil. À ce propos, elle identifie trois catégories de migrants. Elle oppose d'abord les observateurs aux innovateurs volontaires. Les observateurs interagissent essentiellement avec des co-nationaux, si bien que l'exposition à de nouvelles normes passe quasi exclusivement par les médias et l'observation des pratiques et des mœurs dans leur société d'accueil. Leurs comportements sont de fait peu altérés par l'expérience migratoire. Les innovateurs volontaires, en revanche, cherchent activement dans leur société d'accueil des nouvelles idées et expériences. Leur expérience migratoire génère donc des normes sociales hybrides qui combinent leurs normes originelles à celles acquises en migration. Levitt (1998) distingue enfin un troisième groupe d'individus qui se situent à mi-chemin des deux précédents ${ }^{7}$ et qui, en s'adaptant à leur environnement, adoptent de nouvelles normes sans que leurs normes originelles ne soient vraiment altérées. L'acquisition de nouvelles normes lors de l'expérience migratoire ne va donc pas de soi et apparaît à des degrés variés en fonction des interactions que les migrants développent avec leur société d'accueil. Il peut également arriver que la migration entraine un repli identitaire et un rejet des valeurs du pays d'accueil. Il est donc important de tenter d'apprécier l'ampleur et les conditions de l'adoption de normes par les migrants.

Dans un travail récent publié dans le journal Comparative Migration Studies, ${ }^{8}$ nous explorons l'influence de l'expérience migratoire sur la

6. Respectivement les "recipient observers" et les "purposeful innovators » dans la terminologie de Levitt (1998).

7. Elle les qualifie de "instrumental adapters ».

8. Chauvet et al. (2016). 
perception des institutions et les attitudes politiques de migrants maliens vivant en France et en Côte d'Ivoire. À notre connaissance, il n'existe pas d'analyses quantitatives de ce phénomène pour les migrants africains. Dans le cas mexicain, Pérez-Amendáriz et Crow (2010) mettent en évidence, à partir d'entretiens auprès d'un échantillon de 650 citoyens en âge de voter, que les individus ayant vécu aux États-Unis ou au Canada sont plus critiques à l'égard du gouvernement mexicain. Ils ne trouvent toutefois pas de différences notables dans les taux de participation politique des migrants de retour et de leurs concitoyens. Une analyse similaire a été menée par Rother (2009) sur les migrants philippins. Lauteur compare des migrants philippins de retour de pays de destination démocratiques ou autocratiques à des migrants philippins en attente de départ. ${ }^{9}$ L'analyse de Rother suggère que les migrants accordent plus ou moins d'importance aux valeurs démocratiques, aux libertés et aux droits du travail en fonction du type d'institutions politiques prévalant dans les pays les ayant accueillis. Enfin, dans le cas des pays d'Europe de l'Est et Centrale, Careja et Emmenegger (2012) mettent en évidence que les migrants rentrant de pays d'Europe de l'Ouest ont des attitudes politiques différentes des individus n'ayant jamais vécu à l'étranger. Leur analyse suggère que les migrants et les non-migrants ne présentent pas de différences dans la façon dont ils apprécient la vie politique dans leur pays d'origine. En revanche, ces deux groupes d'individus diffèrent du point de vue de leur participation politique et de l'intérêt qu'ils portent aux affaires de l'Union européenne et aux questions de politique étrangère. Il semble notamment que les migrants de retour soient plus impliqués politiquement que les non-migrants. Par ailleurs, de façon cohérente avec les conclusions de Rother dans le cas des Philippines, Careja et Emmeneger soulignent l'importance du type d'institutions politiques dans le pays d'accueil, les différences de participation politique entre migrants et non-migrants apparaissant exclusivement dans le cas de la migration vers des pays démocratiques.

Dans la lignée de ces travaux, nous avons étudié l'influence de l'expérience migratoire sur la perception des institutions et les attitudes politiques de migrants maliens installés en France et en Côte d'Ivoire. Ces

9. Les enquêtés ont tous signé un contrat en tant que Overseas Filipino Worker (OFW), ce qui présente l'immense avantage de pouvoir comparer des migrants de retour et des individus en partance, ayant donc des caractéristiques individuelles très proches mais ayant été exposés, ou non, à des institutions politiques différentes de celles des Philippines. 
deux pays sont les principales destinations des migrants maliens en Europe et en Afrique respectivement. Notre analyse repose sur des données d'enquêtes originales collectées lors du premier tour de l'élection présidentielle malienne en juillet $2013^{10}$. L'originalité de ces données tient dans le fait qu'elles ont été collectées le même jour, auprès de votants maliens, dans trois pays différents : la France, la Côte d'Ivoire et le Mali. En France, les enquêteurs étaient présents sur trois sites franciliens : Bagnolet, Montreuil et Évry. L'enquête en Côte d'Ivoire s'est déroulée dans trois communes du nord d'Abidjan : Abobo, Adjamé et Attécoubé ${ }^{11}$. Enfin, l'enquête au Mali couvrait les communes I, IV et VI de Bamako ainsi que trois communes de la région de Kayes : Kayes, Kenieba et Kita ${ }^{12}$. La région de Kayes a été retenue pour l'enquête car elle est, pour des raisons historiques, la principale zone de départ des migrants maliens vers la France. Notre échantillon final se compose de 188 Maliens en France, 225 en Côte d'Ivoire et 658 au Mali (répartis à peu près également entre Bamako et Kayes).

Nous avons conçu ces enquêtes multi-situées de manière à être parfaitement comparables entre elles. Le tableau 1 présente les différentes mesures auxquelles nous avons recours pour saisir la perception qu'ont les individus des institutions maliennes ainsi que leur intérêt pour la vie politique de leur pays. Il semble que, de façon générale, les Maliens vivant en Côte d'Ivoire (colonne 3) ont une plus grande confiance dans les institutions maliennes que les individus n'ayant jamais migré (colonne 1). ${ }^{13}$ L'inverse apparaît dans le cas des Maliens vivant en France (colonne 2), ces derniers exprimant plus de scepticisme à l'égard des institutions maliennes que les non-migrants. Les questions portant sur l'intérêt des individus pour la vie politique malienne suggèrent que les migrants s'y intéressent plus que les non-migrants, tout en étant en moyenne moins impliqués dans des partis politiques maliens, reflétant les difficultés qu'entraine l'éloignement dans l'implication politique des individus.

10. Ces données sont issues d'un travail conjoint avec Jean-Philippe Dedieu (IRIS, DIAL). Ces enquêtes ont été financées par l'Agence national de la recherche française (ANR) via le programme de recherche POLECOMI [https://dial.ird.fr/projets-de-recherche/projets-anr/polecomi].

11. La collecte à Abidjan a été supervisée par Anda David et Marion Mercier (DIAL).

12. La collecte au Mali a été supervisée par Arouna Sougane (INSTAT).

13. À l'exception de la confiance dans les médias et l'Armée. 
Tableau 1. Degré de confiance dans la démocratie malienne et intérêt pour la politique, non-migrants, migrants en France et en Côte d'Ivoire, 2013 (en \%)

\begin{tabular}{|c|c|c|c|c|}
\hline & $\begin{array}{l}\text { Non- } \\
\text { migrants }\end{array}$ & $\begin{array}{l}\text { Migrants } \\
\text { en France }\end{array}$ & $\begin{array}{c}\text { Migrants } \\
\text { en Côte } \\
\text { d'Ivoire }\end{array}$ & Tous \\
\hline & (1) & (2) & (3) & (4) \\
\hline \multicolumn{5}{|l|}{ Confiance dans $($ Oui $=1)$} \\
\hline la démocratie malienne & 21 & 24 & 43 & 26 \\
\hline $\begin{array}{l}\text { les hommes politiques } \\
\text { maliens (avant le coup) }\end{array}$ & 13 & 12 & 28 & 16 \\
\hline $\begin{array}{l}\text { les hommes politiques } \\
\text { maliens (après le coup) }\end{array}$ & 34 & 15 & 37 & 32 \\
\hline la justice malienne & 17 & 10 & 31 & 19 \\
\hline la police malienne & 27 & 16 & 34 & 27 \\
\hline les medias maliens & 31 & 22 & 28 & 29 \\
\hline l'armée malienne & 43 & 26 & 33 & 38 \\
\hline \multicolumn{5}{|l|}{ Intérêt pour (Oui = 1) } \\
\hline la vie politique malienne & 36 & 56 & 45 & 42 \\
\hline $\begin{array}{l}\text { l'élection présidentielle } \\
\text { malienne de } 2013\end{array}$ & 72 & 80 & 56 & 70 \\
\hline $\begin{array}{l}\text { Proche d'un parti poli- } \\
\text { tique malien (Oui = 1) }\end{array}$ & 59 & 56 & 58 & 58 \\
\hline $\begin{array}{l}\text { Membre d'un parti poli- } \\
\text { tique malien (Oui }=1 \text { ) }\end{array}$ & 33 & 27 & 21 & 30 \\
\hline Nombre d'observations & 658 & 188 & 225 & 1070 \\
\hline
\end{tabular}

Source : Chauvet et al., 2016.

Champs : les migrants sont des personnes de 18 ans et plus qui résident en France ou en Côte d'Ivoire depuis au moins six mois. Les non-migrants sont des individus de 18 ans et plus qui n’ont jamais vécu plus de 6 mois en dehors du Mali. 
Afin d'aller au-delà de ces statistiques descriptives, nous procédons à une analyse multivariée pour évaluer l'impact de l'expérience migratoire (être un migrant en France ou en Côte d'Ivoire) sur chacun des indicateurs du tableau 1. Cette analyse soulève la question délicate du traitement statistique de l'endogénéité du statut migratoire, puisqu'il est en effet possible que les individus décidant de partir vivre à l'étranger soient motivés dans leur choix par des raisons politiques, une appétence accrue pour la démocratie par exemple. Dans ce cas, la corrélation entre l'expérience migratoire et les comportements politiques reflèterait ces préférences politiques plutôt que l'acquisition de normes au cours de l'expérience migratoire.

Au niveau méthodologique, cela implique de recourir à une stratégie économétrique qui tienne compte du fait que le choix de partir en migration dépend parfois des préférences politiques des individus ou de certaines caractéristiques qui sont elles-mêmes corrélées à leurs préférences politiques, telles que l'éducation. Pour cela, il faut trouver une source de variation dans la probabilité de partir en migration qui soit exogène aux préférences politiques des individus. ${ }^{14}$ Nous nous appuyons sur des caractéristiques macro-économiques des pays de destination, qui expliquent largement l'attrait de ces destinations pour les Maliens, tout en étant peu corrélées avec leurs perceptions et attitudes politiques. Pour expliquer la migration vers la France, nous utilisons, pour chaque individu, le taux de croissance annuel du produit intérieur brut français - qui saisit la demande de travail en France - l'année de ses 24 ans (l'âge moyen de départ en migration dans notre échantillon). De façon similaire, pour expliquer la migration vers la Côte d'Ivoire nous utilisons le nombre annuel de victimes liées au conflit civil à Abidjan - facteur dissuadant les individus de partir.

Nos résultats confirment les statistiques descriptives du tableau 1 : les migrants ont des comportements politiques et une perception des institutions maliennes différentes des non-migrants. Ces différences dépendent du pays dans lequel ils résident. Il apparaît en effet que les Maliens vivant en France perçoivent de manière plus critique les institutions maliennes que les non-migrants, alors que les perceptions des Maliens vivant en Côte d'Ivoire ne sont pas significativement différentes de celles des non-migrants. Ces résultats suggèrent que l'acquisition de normes politiques lors de l'expérience

14. Par ailleurs, nos estimations incluent les caractéristiques suivantes : âge, sexe, groupe ethnique, région de naissance, éducation, appartenance au conseil de village ou à l'élite religieuse locale. 
migratoire dépend des normes qui prévalent dans les pays d'origine et de destination. Toutefois, les interactions que les migrants ont avec leur société d'accueil peuvent également intervenir dans le processus. Pour évaluer l'importance de ce dernier facteur, nous examinons l'incidence du degré de socialisation des migrants maliens en France et en Côte d'Ivoire sur la façon dont ils perçoivent les institutions politiques maliennes (tableaux 2 et 3 ).

Le degré de socialisation des migrants dans chacun des deux pays d'accueil est appréhendé à travers leur utilisation des médias (français ou ivoiriens) comme source d'information, leur niveau d'éducation, leur appartenance à un syndicat (français ou ivoirien) et enfin, pour le cas de la France, leur cadre de vie - selon qu'ils vivent en foyers de travailleurs immigrés ou bien en appartement individuel. Les statistiques descriptives présentées dans les tableaux 2 et 3 suggèrent que le scepticisme des migrants installés en France vis-à-vis des institutions de leur pays d'origine est plus fort lorsqu'ils sont consommateurs de médias français, ont poursuivi des études supérieures et appartiennent à un syndicat. La situation est assez similaire dans le cas des Maliens de Côte d'Ivoire, même si la faiblesse des effectifs dans certaines catégories impose une certaine prudence.

Cet ensemble de résultats semble indiquer que la migration est un vecteur potentiel d'adoption de normes et pratiques politiques nouvelles mais que l'influence de l'expérience migratoire dépend de manière critique des caractéristiques du pays de destination et du degré de socialisation et d'intégration des migrants dans leur société d'accueil. 
Tableau 2. Degré de confiance dans la démocratie malienne et intérêt pour la vie politique des non migrants et des migrants en France, selon leur degré de socialisation en France, 2013 (en \%)

\begin{tabular}{|c|c|c|c|c|c|c|c|}
\hline \multirow[b]{3}{*}{$\begin{array}{l}\text { Confiance } \\
\text { dans }(\text { Oui }=1)\end{array}$} & \multirow{3}{*}{$\begin{array}{c}\text { Non } \\
\text { migrants }\end{array}$} & \multicolumn{6}{|c|}{ Migrants } \\
\hline & & \multicolumn{2}{|c|}{$\begin{array}{l}\text { Éducation } \\
\text { supérieure }\end{array}$} & \multicolumn{2}{|c|}{$\begin{array}{c}\text { Membre } \\
\text { d'un syndicat }\end{array}$} & \multicolumn{2}{|c|}{$\begin{array}{l}\text { Vit dans } \\
\text { un foyer }\end{array}$} \\
\hline & & Oui & Non & Oui & Non & Oui & Non \\
\hline $\begin{array}{l}\text { la démocratie } \\
\text { malienne }\end{array}$ & 21 & 4 & 29 & 27 & 23 & 24 & 25 \\
\hline $\begin{array}{l}\text { les hommes } \\
\text { politiques ma- } \\
\text { liens (avant le } \\
\text { coup) }\end{array}$ & 13 & 4 & 15 & 15 & 12 & 21 & 11 \\
\hline $\begin{array}{l}\text { les hommes } \\
\text { politiques ma- } \\
\text { liens (après le } \\
\text { coup) }\end{array}$ & 35 & 12 & 17 & 13 & 19 & 11 & 18 \\
\hline $\begin{array}{l}\text { la justice } \\
\text { malienne }\end{array}$ & 18 & 0 & 13 & 10 & 11 & 16 & 9 \\
\hline $\begin{array}{l}\text { la police } \\
\text { malienne }\end{array}$ & 28 & 4 & 15 & 05 & 19 & 16 & 13 \\
\hline $\begin{array}{l}\text { les medias } \\
\text { maliens }\end{array}$ & 31 & 4 & 25 & 16 & 26 & 27 & 20 \\
\hline $\begin{array}{l}\text { l'armée } \\
\text { malienne }\end{array}$ & 43 & 23 & 24 & 15 & 31 & 34 & 21 \\
\hline $\begin{array}{l}\text { Nombre } \\
\text { d'observations }\end{array}$ & 673 & 27 & 146 & 74 & 99 & 38 & 135 \\
\hline
\end{tabular}


Tableau 3. Degré de confiance dans la démocratie malienne et intérêt pour la vie politique des non migrants et des migrants en Côte d'Ivoire, selon leur degré de socialisation en Côte d'Ivoire, 2013 (en \%)

\begin{tabular}{|l|c|c|c|c|c|}
\cline { 2 - 6 } \multicolumn{1}{l|}{} & $\begin{array}{c}\text { Non } \\
\text { migrants }\end{array}$ & \multicolumn{4}{c|}{ Migrants } \\
\cline { 2 - 6 } & & $\begin{array}{c}\text { Éducation } \\
\text { supérieure }\end{array}$ & \multicolumn{2}{c|}{$\begin{array}{c}\text { Membre } \\
\text { d'un syndicat }\end{array}$} \\
\hline $\begin{array}{l}\text { Confiance dans } \\
\text { (Oui=1) }\end{array}$ & Oui & Non & Oui & Non \\
\hline $\begin{array}{l}\text { la démocratie } \\
\text { malienne }\end{array}$ & 13 & 0 & 29 & 8 & 28 \\
\hline $\begin{array}{l}\text { les hommes } \\
\text { politiques maliens } \\
\text { (avant le coup) }\end{array}$ & 35 & 33 & 37 & 17 & 39 \\
\hline $\begin{array}{l}\text { les hommes } \\
\text { politiques maliens } \\
\text { (après le coup) }\end{array}$ & 18 & 7 & 30 & 8 & 30 \\
\hline la justice malienne & 28 & 13 & 35 & 25 & 34 \\
\hline la police malienne & 31 & 13 & 27 & 8 & 27 \\
\hline les medias maliens & 43 & 7 & 33 & 17 & 32 \\
\hline l'armée malienne & $\mathbf{6 7 3}$ & $\mathbf{1 5}$ & $\mathbf{1 9 3}$ & $\mathbf{1 2}$ & $\mathbf{1 9 5}$ \\
\hline $\begin{array}{l}\text { Nombre } \\
\text { d'observations }\end{array}$ & & & & \\
\hline
\end{tabular}

Source : Chauvet et al., 2016.

Champs : les migrants sont des personnes de 18 ans et plus qui résident en France ou en Côte d'Ivoire depuis au moins six mois. Les non-migrants sont des individus de 18 ans et plus qui n'ont jamais vécu plus de six mois en dehors du Mali. 


\section{Migration de retour et transfert de normes politiques}

Alors que nous venons d'observer que l'expérience migratoire modifie sensiblement la perception qu'ont les migrants maliens des institutions politiques de leur pays d'origine, il convient d'examiner si les migrants conservent durablement ces normes adoptées en migration et sont à même d'influencer les opinions voire les pratiques de leurs proches une fois rentrés dans leur pays d'origine.

En effet, la persistance des "bénéfices " tirés par les migrants de retour de leur expérience migratoire (the "returns of returning ") dépend de plusieurs facteurs. Si les opinions des migrants sont conditionnées par l'environnement dans lequel ils vivent, il est possible qu'une fois de retour ces dernières changent et convergent vers celles des non-migrants. Il peut en être de même si les migrants de retour cessent d'avoir des contacts avec la diaspora et leurs réseaux dans le pays d'émigration, ou bien s'ils ont moins accès aux médias du pays d'émigration. Par ailleurs, ceux qui rentrent sont potentiellement des personnes ayant rencontré des difficultés d'insertion dans la société du pays d'accueil, ou dont l'expérience migratoire a été un échec, ressenti ou réel. Il peut en résulter un rejet vis-à-vis des valeurs de la société d'accueil.

Ensuite, au-delà de l'adoption durable de nouvelles normes par les migrants, il convient d'examiner si, une fois de retour, les migrants partagent leurs opinions et exercent une influence sur les pratiques sociales et politiques de leur entourage. La diffusion des normes acquises en migration à la communauté d'origine dépend de l'écoute que reçoivent les migrants de retour ainsi que de leur rôle de leaders. Cela renvoie à leur statut économique et social au sein de leur communauté, ainsi qu'aux conditions de leur retour aux pays (subi ou volontaire), au contexte politique et social de leur pays d'origine et aux écarts existant entre leurs opinions et celles des non-migrants.

Pour tenter de répondre à ces questions dans le contexte malien, nous avons mené différents travaux. Dans un premier travail nous examinons le transfert de normes politiques au sein du ménage (Chauvet et al., 2017), tandis que dans un second travail nous analysons le transfert de normes politiques au niveau des communes (Chauvet et Mercier, 2014). 


\subsection{Migrants de retour, intérêts et implications dans la vie politique malienne}

Un premier travail porte sur l'intérêt des migrants de retour pour la vie politique malienne, ainsi qu'à la diffusion des normes politiques acquises en migration à leurs familles (Chauvet et al., 2017). Sur la base d'une enquête conduite au cours de l'année 2013 auprès de 5300 ménages, soit environ 15000 individus de 18 ans et plus ${ }^{15}$, cette recherche examine tout d'abord si les migrants de retour participent différemment à la vie politique malienne que les non-migrants.

À ce titre, l'enquête de 2013 est riche d'enseignements. Il ressort que $52 \%$ des migrants de retour expriment beaucoup ou un peu d'intérêt pour la politique malienne ${ }^{16}$ contre seulement $37 \%$ des non-migrants. L'écart entre les deux groupes est de même ampleur lorsqu'il s'agit de l'intérêt porté à la campagne présidentielle de 2013 : $42 \%$ des migrants de retour s’y sont intéressés contre $27 \%$ des non-migrants. Les migrants de retour apparaissent aussi plus impliqués politiquement que les non-migrants : ils sont $20 \%$ à affirmer avoir participé activement à la campagne présidentielle de 2013, $59 \%$ à se sentir proches d'un parti politique et $18 \%$ à être membres d'un parti, contre seulement respectivement $10 \%, 45 \%$ et $9 \%$ des non-migrants. Les migrants de retour semblent également plus investis localement que les non-migrants. La moitié est membre d'une association et assiste aux réunions du conseil de village ou de quartier, contre respectivement $31 \%$ et $15 \%$ des non-migrants. Enfin, ils sont presque $10 \%$ à participer aux conseils municipaux contre $2 \%$ des non-migrants.

15. Cette enquête a été menée en partenariat avec l'Institut malien de la statistique (INSTAT) et a été financée par l'Agence national de la recherche française (ANR) via le programme de recherche POLECOMI [https://dial.ird.fr/projets-de-recherche/projets-anr/ polecomi] et par l'Union européenne à travers le programme Nopoor [http://www.nopoor. eu/](FP7 Framework Programme for Research of the European Union -SSH.2011.4.1-1 : Tackling poverty in a development context, Collaborative project/Specific International Cooperation Action. Grant Agreement No. 290752).

16. Les autres modalités de réponse à cette question sont : " non, pas vraiment " et " non, pas du tout ». 


\section{Tableau 4. Intérêt pour et implication dans la vie politique,} migrants de retour vs. non migrants, Mali, 2013 (en \%)

\begin{tabular}{|l|c|c|c|}
\cline { 2 - 4 } \multicolumn{1}{l|}{} & $\begin{array}{c}\text { (1) } \\
\text { Migrants } \\
\text { de retour }\end{array}$ & $\begin{array}{c}\text { Non } \\
\text { migrants }\end{array}$ & $\begin{array}{c}\text { Différence } \\
\text { entre (1) et (2) }\end{array}$ \\
\hline $\begin{array}{l}\text { Est intéressé par la } \\
\text { politique malienne }\end{array}$ & 51,8 & 36,8 & $0,149^{* * *}$ \\
\hline $\begin{array}{l}\text { Est intéressé par la } \\
\text { campagne présidentielle } \\
\text { de 2013 }\end{array}$ & 41,6 & 27,5 & $0,141^{* * *}$ \\
\hline $\begin{array}{l}\text { Participe activement à la } \\
\text { campagne prés. de 2013 }\end{array}$ & 19,7 & 10,5 & $0,091^{* * *}$ \\
\hline $\begin{array}{l}\text { Est proche d'un parti } \\
\text { politique }\end{array}$ & 59,4 & 44,7 & $0,147^{* * *}$ \\
\hline $\begin{array}{l}\text { Est membre d'un parti } \\
\text { politique }\end{array}$ & 17,9 & 8,9 & $0,090^{* * *}$ \\
\hline $\begin{array}{l}\text { Est membre d'une } \\
\text { association }\end{array}$ & 50,5 & 31,2 & $0,193^{* * *}$ \\
\hline $\begin{array}{l}\text { Participe aux réunions du } \\
\text { conseil de village/quartier } \\
\text { municipaux }\end{array}$ & $\mathbf{1 3 3 1}$ & $\mathbf{1 3 3 5 5}$ & $\mathbf{1 4} \mathbf{6 8 6}$ \\
\hline Nombre d'observations & 2,5 & $0,073^{* * *}$ \\
\hline
\end{tabular}

Source : EMOP, 2013, calcul des auteures.

Champs : les migrants de retour sont des personnes de 18 ans et plus qui résident en 2013 
au Mali et qui ont séjourné au moins six mois dans un pays autre que le Mali au cours de leur vie. Les non-migrants sont des individus de 18 ans et plus qui n'ont jamais vécu plus de six mois en dehors du Mali et qui ne vivent pas dans un ménage avec au moins un migrant de retour. Sont aussi exclus de l'échantillon des non-migrants les individus qui vivent dans un ménage avec au moins une personne en migration en 2013.

En colonne (3), les étoiles représentent la significativité statistique des différences entre les pourcentages des colonnes (1) et (2), ${ }^{* *} \mathrm{p}<0,01 ;{ }^{* *} \mathrm{p}<0,05 ;{ }^{*} \mathrm{p}<0,1$.

Cependant, ces écarts peuvent provenir du fait que les migrants de retour ont, indépendamment de leur expérience à l'étranger, des caractéristiques individuelles qui les distinguent de celles des non-migrants. Toutefois, ces caractéristiques spécifiques sont susceptibles d'influencer l'intérêt pour la vie politique dans un sens ou dans l'autre, si bien qu'il est difficile de conclure quant à leur influence sur la relation étudiée. Le tableau 5 montre en effet que les migrants sont quasi exclusivement des hommes (87,4\%) et qu'ils sont plus âgés, ce qui laisse présager d'un intérêt et d'une implication plus forts dans la vie politique malienne. Cependant, le fait qu'ils soient moins éduqués, qu'ils résident moins dans la capitale et plus dans les régions frontalières (région de Kayes et de Sikasso) et qu'ils soient moins nombreux à avoir pour langue maternelle la principale langue parlée du Mali (le bambara) sont des éléments qui, au contraire, pourraient être à l'origine d'une moindre présence des migrants de retour dans les instances politiques. 
Tableau 5. Caractéristiques sociodémographiques, migrants de retour vs. non-migrants, Mali, 2013 (en \%)

\begin{tabular}{|l|c|c|c|}
\cline { 2 - 4 } \multicolumn{1}{|c|}{} & $\begin{array}{c}(\mathbf{1}) \\
\text { Migrants de } \\
\text { retour }\end{array}$ & $\begin{array}{c}\text { (2) } \\
\text { Non } \\
\text { migrants }\end{array}$ & $\begin{array}{c}\text { (3) } \\
\text { Différence } \\
\text { entre (1) et (2) }\end{array}$ \\
\hline Hommes & 87,4 & 47,9 & $0,395^{* * *}$ \\
\hline Âge moyen & 47,3 & 36,5 & $10,885^{* * *}$ \\
\hline Niveau d'éducation & \multicolumn{3}{|c|}{} \\
\hline Sans éducation & 78,2 & 69,3 & $0,089^{* * *}$ \\
\hline Primaire & 17,1 & 19,7 & $-0,026^{*}$ \\
\hline Secondaire & 2,3 & 8,0 & $-0,058^{* * *}$ \\
\hline Tertiaire & 2,4 & 3,0 & $-0,005$ \\
\hline Région de résidence & \multicolumn{3}{|c|}{} \\
\hline Kayes & 15,5 & 10,1 & $0,054^{* * *}$ \\
\hline Koulikoro & 7,7 & 24,0 & $-0,164^{* * *}$ \\
\hline Sikasso & 24,9 & 13,5 & $0,114^{* * *}$ \\
\hline Ségou & 13,8 & 20,9 & $-0,070^{* * *}$ \\
\hline Mopti & 27,7 & 10,0 & $0,178^{* * *}$ \\
\hline Bamako & 10,4 & 21,5 & $-0,111^{* * *}$ \\
\hline Langue maternelle & 43,9 & 57,6 & $-0,137^{* * *}$ \\
\hline bambara & $\mathbf{1 3 3 1}$ & $\mathbf{1 3 3 5 5}$ & $\mathbf{1 4 6 8 6}$ \\
\hline Observations & \multicolumn{2}{|c|}{} \\
\hline
\end{tabular}

Source : EMOP, 2013, calcul des auteures. 
Champs : les migrants de retour sont des personnes de 18 ans et plus qui résident en 2013 au Mali et qui ont séjourné au moins six mois dans un pays autre que le Mali au cours de leur vie. Les non-migrants sont des individus de 18 ans et plus qui n’ont jamais vécu plus de six mois en dehors du Mali et qui ne vivent pas dans un ménage avec au moins un migrant de retour. Sont aussi exclus de l'échantillon des non-migrants les individus qui vivent dans un ménage avec au moins une personne en migration en 2013.

En colonne (3), les étoiles représentent la significativité statistique des différences entre les pourcentages des colonnes (1) et (2), ${ }^{* * *} \mathrm{p}<0,01 ;{ }^{* *} \mathrm{p}<0,05 ;{ }^{*} \mathrm{p}<0,1$.

Au-delà de leurs caractéristiques individuelles, la littérature suggère que l'intérêt des migrants de retour pour la vie politique malienne dépend des caractéristiques politiques des pays dans lesquels ils ont vécu. Les migrants en provenance de pays démocratiques apparaissent en effet significativement plus intéressés par la politique malienne, et plus précisément par la campagne présidentielle de 2013, que les migrants en provenance de pays moins démocratiques (Chauvet et al., 2017). De même, ils se sentent plus proches d'un parti politique, et participent activement aux réunions des conseils villageois et de quartier que les non-migrants ou que les migrants en provenance de pays moins démocratiques. Par ailleurs, ces derniers sont plus actifs politiquement que les non-migrants. Les tests statistiques montrent qu'ils ont participé plus activement à la campagne électorale de 2013 et sont plus enclins que les non-migrants à être membres d'une association, voire à être membres d'un parti politique et à participer aux conseils municipaux. L'expérience migratoire semble donc bien être un facteur de mobilisation politique, et son influence s'amplifie avec le degré de démocratie dans le pays d'accueil.

\subsection{L'intérêt politique des migrants de retour se diffuse-il au sein de leurs familles?}

S'il semble que les migrants de retour présentent un intérêt plus vif pour la vie politique malienne, qu'en est-il de leur entourage familial ? Estce que les migrants de retour diffusent leurs normes politiques à leur famille ? Des éléments de réponse peuvent être trouvés dans la comparaison des individus qui vivent dans un ménage avec et sans migrant de retour. Le tableau 6 illustre cette comparaison. 
Tableau 6. Intérêt et participations politiques, individus non migrants vivant dans des ménages avec ou sans migrants de retour, Mali, 2013 (en \%)

\begin{tabular}{|c|c|c|c|}
\hline & $\begin{array}{c}\text { (1) } \\
\text { Individus } \\
\text { vivant dans } \\
\text { des ménages } \\
\text { avec migrants } \\
\text { de retour }\end{array}$ & $\begin{array}{c}(2) \\
\text { Individus } \\
\text { vivant } \\
\text { dans des } \\
\text { ménages } \\
\text { sans } \\
\text { migrants }\end{array}$ & $\begin{array}{c}\text { (3) } \\
\text { Différence } \\
\text { entre } \\
\text { (1) et (2) }\end{array}$ \\
\hline $\begin{array}{l}\text { Est intéressé par la } \\
\text { politique malienne }\end{array}$ & 30,0 & 36,8 & $-0.068^{* * *}$ \\
\hline $\begin{array}{l}\text { Est intéressé par la } \\
\text { campagne présidentielle } \\
\text { de } 2013\end{array}$ & 31,3 & 27,5 & $0.038^{*}$ \\
\hline $\begin{array}{l}\text { Participe activement à la } \\
\text { campagne prés. de } 2013\end{array}$ & 9,6 & 10,5 & -0.009 \\
\hline $\begin{array}{l}\text { Est proche d'un parti } \\
\text { politique }\end{array}$ & 56,8 & 44,7 & $0.121^{* * *}$ \\
\hline $\begin{array}{l}\text { Est membre d'un parti } \\
\text { politique }\end{array}$ & 8,5 & 8,9 & -0.004 \\
\hline $\begin{array}{l}\text { Est membre d'une } \\
\text { association }\end{array}$ & 44,6 & 31,2 & $0.134^{* * *}$ \\
\hline $\begin{array}{l}\text { Participe aux réunions du } \\
\text { conseil de village/quartier }\end{array}$ & 9,8 & 15,3 & $-0.056^{* * *}$ \\
\hline $\begin{array}{l}\text { Participe aux conseils } \\
\text { municipaux }\end{array}$ & 2,2 & 2,5 & -0.003 \\
\hline Observations & 4953 & 13355 & 18308 \\
\hline
\end{tabular}

Source : EMOP, 2013, calcul des auteures 
Champs : personnes âgées de 18 ans et plus qui n’ont jamais vécu plus de 6 mois en dehors du Mali qui vivent soit dans un ménage avec au moins un migrant de retour (colonne 1), soit qui ne vivent pas dans un ménage avec au moins un migrant de retour. Sont exclus de cet échantillon les individus qui vivent dans un ménage avec au moins une personne en migration en 2013.

Des différences apparaissent. Par exemple $45 \%$ des individus vivant dans des ménages avec au moins un migrant de retour participent à une association, contre seulement $31 \%$ des individus vivant dans des ménages sans migrant. De même, ils se sentent plus proches d'un parti politique malien ( $57 \%$ contre $45 \%$ ) et sont plus intéressés par la campagne présidentielle de 2013 (31\% contre $27 \%$ ). Cependant, d'autres indicateurs ne sont pas significativement différents, voire sont plus faibles, comme l'intérêt porté à la vie politique malienne.

La prise en compte des autres caractéristiques individuelles de ces deux groupes permet d'affiner le diagnostic. Nous observons alors un transfert de normes politiques des migrants de retour à leurs familles. Les individus vivant dans des ménages avec migrants de retour en provenance de pays démocratique expriment notamment un intérêt significativement plus marqué pour la campagne présidentielle de 2013 et participent plus à la vie associative et politique locale.

\subsection{Diffusion de normes politiques par les migrants de retour au sein de leur village}

Il est possible que le processus de transfert de normes politiques s'opère au-delà du cercle familial, par exemple à l'échelle des villages d'origine des migrants. En effet, la littérature a mis en évidence qu'au Mexique, en Moldavie, au Maroc ou au Mozambique, les individus résidant dans des régions concentrant un nombre important de migrants de retour avaient des comportements électoraux différents de ceux vivant dans des régions moins tournées vers l'extérieur.

Dans le cas du Mali, les résultats électoraux des derniers scrutins municipaux ont été mis en rapport avec le pourcentage de migrants de retour au sein de chaque commune. Les résultats de ce travail sont présentés en détail dans Chauvet et Mercier (2014) qui analysent l'influence de la migration de retour sur les taux de participation et la concurrence électorale lors des élections municipales maliennes. Cette étude mobilise des données 
de recensement (1998 et 2009) agrégées au niveau des 700 communes maliennes et combinées aux résultats des élections municipales de 1998 et 2009. La concurrence électorale est mesurée par une variable de fragmentation des votes, ainsi que par une variable d'écart de victoire entre les deux listes ayant obtenu le plus de voix. Les données de recensement permettent de calculer la part du stock de migrants de retour dans la population de chaque commune.

Les résultats de cette étude suggèrent que la migration de retour tend à augmenter la participation aux élections et, de manière un peu moins robuste, la concurrence du processus électoral. Cependant, un effet significativement positif du stock de migrants de retour sur le taux de participation n'atteste pas directement d'un transfert de normes politiques. Il peut ne refléter qu'une participation accrue des seuls migrants de retour. Par une simulation simple, nous montrons toutefois que la présence de migrants de retour dans la localité augmente le taux de participation au-delà de leur propre participation, ce qui suggère un transfert de normes vers la communauté dans son ensemble. Ces résultats semblent essentiellement reposer sur l'influence des migrants qui rentrent de pays autres que ceux situés sur le continent africain. Cette étude semble donc indiquer que les migrants de retour en provenance de pays démocratiques insufflent dans leur commune de résidence une dynamique électorale différente de celle des autres communes maliennes.

\section{Conclusion}

L'ensemble des travaux que nous avons menés sur les migrants maliens suggère que l'expérience migratoire influence la façon dont ils perçoivent les institutions démocratiques maliennes ainsi que leur intérêt pour la vie politique de leur pays d'origine. L'environnement politique, comme leur mode d'insertion dans le pays d'accueil, contribuent à modeler leur perception de la vie politique malienne, et ce de manière durable puisque de retour au Mali leurs perceptions demeurent différentes de celles des non-migrants.

Peut-on pour autant espérer que les migrants soient des vecteurs de consolidation des institutions démocratiques maliennes ? Nos travaux suggèrent que les migrants de retour tendent à diffuser les normes politiques acquises en migration à leur famille d'origine, et plus largement à leur communauté. La mobilisation des migrants semble donc constituer un 
levier intéressant de changement politique. Cependant, les migrants les plus critiques et potentiellement les plus « armés " pour insuffler ce changement, c'est-à-dire essentiellement ceux en provenance des pays démocratiques ne sont qu'une minorité parmi ceux qui ont choisi de revenir vivre au Mali.

\section{RÉFÉRENCES BIBLIOGRAPHIQUES}

Batista, C. et Vicente, P. C., 2011, "Do migrants improve governance at home? Evidence from a voting experiment", The World Bank Economic Review 25 (1), 77-104.

Batista, C., Seither, J. et Vicente, P. C., 2017, «Les réseaux de migrants et les transferts de normes politiques au Mozambique et au Cap-Vert ", chapitre 5 in Chauvet, Gubert, Jaulin et Mesplé-Somps. Les migrants, acteurs du changement politique en Afrique?, De Boeck Supérieur.

Barsbai, T., Rapoport, H., Steinmayr, A. et Trebesch, C., 2017, “The Effect of Labor Migration on the Diffusion of Democracy: Evidence from a Former Soviet Republic", American Economic Journal: Applied Economics, forthcoming.

Beine, M., Docquier, F. et Schiff, M., 2013, "International Migration, Transfers of Norms and Home Country Fertility", Canadian Journal of Economics, 46 (4), 1406-1430.

Boilley, P., 1999, Les Touaregs Kel Adagh : dépendance et révoltes : du Soudan français au Mali contemporain, Paris, Karthala.

Bourgeot, A., 1996, "Les rébellions touaregs : une cause perdue? ", Afrique contemporaine, $\mathrm{n}^{\circ} 182,99-115$.

Bourgeot, A., 2013, «Des Touaregs en rébellion », in P. Gonin, N. Kotlok et M-A. Pérouse De Montclos, La tragédie malienne, Paris, Éd. Vendemiaire, p. 113-130.

Bratton, M. et Gyimah-Boadi, E., 2015, "Political risks facing African democracies: evidence from Afrobarometer", Afrobarometer Working Pa$\operatorname{per} \mathrm{n}^{\circ} 157$. 
Careja. R. \& Emmenegger, P., 2012, "Making Democratic Citizens The Effects of Migration Experience on Political Attitudes in Central and Eastern Europe", Comparative Political Studies 45 (7), 875-902.

Chauvet, L., Gubert, F. et Mesplé-Somps, S., 2013, "Transferts migratoires et démocratisation ", in Gonin P., N. Kotlok et M-A. Pérouse De Montclos, La tragédie malienne, Paris, Éd. Vendemiaire, p. 227-241.

Chauvet, L., Gubert, F. et Mesplé-Somps, S., 2016, "Do migrants adopt new political attitudes from abroad? Evidence using a multi-sited exit-poll survey during the 2013 Malian elections", Comparative Migration Studies, 4 (19).

Chauvet, L., Gubert, F. et Mesplé-Somps, S., 2017, "Are migrants agents of democratic diffusion? Evidence from a Malian case study", mimeo.

Chauvet, L., Mercier, M., 2014, "Do return migrants transfer political nor$\mathrm{ms}$ to their origin country? Evidence from Mali", Journal of Comparative Economics, 42, 630-651.

Docquier, F., Lodigiani, E., Rapoport, H. et Schiff, M., 2011, “Emigration and democracy", IZA Discussion Paper n 5496.

Guichaoua, Y., 2014, “Tuareg Militancy and the Sahelian Shock Waves of the Libyan Revolution", in P.Cole et B. McQuinn, The Libyan Revolution and its Aftermath. Hurst / Oxford University Press.

Guichaoua, Y., 2017, «L'horizon compromis de la force Barkhane au Mali », theconversation.com, https://theconversation.com/lhorizon-compromis-de-la-force-barkhane-au-mali-77340.

Hirschman, A. O., 1970, Exit, voice and loyalty: responses to decline in firms, organizations and states, Harvard University Press, ISBN 0674276604.

Kapur, D., 2010, Diaspora, Development and Democracy, the Domestic Impact of International Migration from India, Princeton University Press, $325 \mathrm{p}$.

Kapur, D. et McHale, J., 2005, Give us your best and brightest: The global hunt for talent and its impact on the developing world, Center for Global Development.

Levitt. P., 1998, "Social remittances: Migration driven local-level forms of cultural diffusion”, International migration review, 926-948. 
Pérez-Armendariz, C. et Crow, D., 2010, "Do migrants remit democracy? International migration, political beliefs, and behavior in Mexico", Comparative political studies 43 (1) : 119-148.

Pfutze, T., 2012, "Does migration promote democratization? Evidence from the Mexican transition", Journal of Comparative Economics, 40 (2) : 159-175.

Pollet, E. et Winter, G., 1971, La société Soninké(Dyahunu, Mali), Éditions de l'Institut de sociologie, Université libre de Bruxelles, Belgique.

Rother, S., 2009, "Changed in migration? Philippine return migrants and (un) democratic remittances", European Journal of East Asian Studies, 8 (2), 245-274.

Sougane, A., 2015, "Migrations et transferts au Mali, un état des lieux ", in J. Charmes, D. Konaté, J. Brunet-Jailly (dir.), Le Mali contemporain, coédition IRD- Éditions Tombouctou, p. 581-612.

Sougane, A., Tounkara, M., Traoré, S. M., Razafindrakoto, M. et Roubaud, F., 2015, Dynamique de la Gouvernance, la paix et la Sécurité au Mali entre 2014 et 2015 : Une analyse à partir des modules GPS-SHaSA, EMOP, INSTAT, octobre 2015.

Spilimbergo, A., 2009, "Democracy and foreign education", American Economic Review, 99 (1) : 528-543.

Tuccio, M. et Wahba, J., 2017, « Changer les opinions politiques grâce aux migrations internationales, l'exemple de l'Afrique du Nord », chapitre 7 in Chauvet, Gubert, Jaulin et Mesplé-Somps, 2017, Les migrants, acteurs du changement politique en Afrique?, De Boeck Supérieur.

Vari-Lavoisier, I., 2017, "Impact de la migration sur la corruption, chapitre 6 " in Chauvet, Gubert, Jaulin et Mesplé-Somps (dir.). Les migrants, acteurs du changement politique en Afrique ? Éditions De Boeck Supérieur. 